\title{
Feasibility of Single-Stage Posterior Passive Correction and Fusion Surgery for Congenital Scoliosis in Adolescent Patients Who Have Attained Skeletal Maturity
}

\author{
Chee Kidd Chiu ${ }^{1}$, Rommel Lim Tan ${ }^{1,2}$, Siti Mariam Abd Gani ${ }^{1}$, Jessamine Sze Lynn Chong ${ }^{1}$, \\ Weng Hong Chung ${ }^{1}$, Chris Yin Wei Chan ${ }^{1}$, Mun Keong Kwan ${ }^{1}$ \\ ${ }^{1}$ Department of Orthopaedic Surgery, National Orthopaedic Centre of Excellence for Research and Learning, Faculty of Medicine, \\ University of Malaya, Kuala Lumpur, Malaysia \\ ${ }^{2}$ Davao Doctors Hospital, Davao City, Philippines
}

Study Design: Retrospective study.

Purpose: To report the perioperative and radiological outcomes of single-stage posterior passive correction and fusion (SSPPCF) in adolescent patients who present with congenital scoliosis.

Overview of Literature: The surgical treatment for congenital scoliosis is complex. There is no definitive guide on surgical options for skeletally matured adolescent patients who have congenital scoliosis.

Methods: Patients with congenital scoliosis who underwent SSPPCF using a pedicle screw system were reviewed. We identified the following three surgical indications: (1) hemivertebra or wedge vertebra over the thoracic or thoracolumbar region with structural lumbar curves, (2) hemivertebra or wedge vertebra at the lumbar region with significant pelvic obliquity or sacral slanting, and (3) mixed or complex congenital scoliosis. The demographic, perioperative, and radiographic data of these patients were collected.

Results: Thirty-four patients were reviewed. The mean patient age was $14.6 \pm 3.4$ years. There were 13 hemivertebrae, three wedged vertebrae, two butterfly vertebrae, three hemivertebrae with butterfly vertebra, eight unsegmented bars, and five multiple complex lesions. The average surgical duration was $219.4 \pm 68.8$ minutes. The average blood loss was $1,208.4 \pm 763.5 \mathrm{~mL}$. Seven patients required allogeneic blood transfusion. The mean hospital stay duration was $6.1 \pm 2.5$ days. The complication rate was $11.8 \%$ (4/34): one patient had severe blood loss, one had rod breakage, and two had distal adding-on. The Cobb angle reduced from $65.9^{\circ} \pm 17.4^{\circ}$ to $36.3^{\circ} \pm 15.3^{\circ}$ $(p<0.001)$ with a correction rate (CR) of $44.8 \% \pm 17.4 \%$. The regional kyphotic angle decreased from $39.9^{\circ} \pm 20.5^{\circ}$ to $27.5^{\circ} \pm 13.9^{\circ}(p=0.001)$ with a CR of $19.3 \% \pm 49.6 \%$. Radiographic parameters (radiographic shoulder height, clavicle angle, T1 tilt, cervical axis, pelvic obliquity, coronal balance, and apical vertebral translation) showed significant improvement postoperatively.

Conclusions: SSPPCF was a feasible option for adolescent patients with congenital scoliosis who were skeletally matured.

Keywords: Congenital; Scoliosis; Adolescent; Spine surgery; Passive correction

Received Dec 20, 2020; Revised Jan 19, 2021; Accepted Jan 24, 2021

Corresponding author: Chee Kidd Chiu

Department of Orthopaedic Surgery, National Orthopaedic Centre of Excellence for Research and Learning, Faculty of Medicine, University of Malaya, 50603 Kuala Lumpur, Malaysia

Tel: +60-379492446, Fax: +60-379494642, E-mail: cheekidd@um.edu.my 


\section{Introduction}

Surgical options for congenital scoliosis vary depending on several factors, such as the type of congenital anomalies, degree of deformity, and age of the patient [1,2]. The following surgical options have been reported: in situ fusion either with posterior spinal fusion (PSF) only or with a combined posterior and anterior spinal fusion [2-6]; convex epiphysiodesis or hemiepiphysiodesis $[7,8]$; expansion thoracoplasty with vertical expandable prosthetic titanium rib implant (VEPTR) $[9,10]$; and growth-guided devices such as growing rod $[4,11]$, pedicle subtraction osteotomy (PSO) [12], hemivertebrectomy [13-15], vertebral column resection (VCR) $[16,17]$, and PSF $[5,15,18]$. In some cases, a combination of multiple procedures was used in a single patient $[7,10]$. Due to the complexity in the choice of surgical management for congenital scoliosis, it is associated with a high risk of neurological and non-neurological complications [19].

After a patient achieved skeletal maturity, surgical options, such as in situ fusion, epiphysiodesis, VEPTR, and growth-guidance devices are less useful or feasible. However, there is still doubt on how to choose the best options for these patients. Procedures involving osteotomies, such as PSO, hemivertebrectomy, and VCR are technically more demanding and have higher complication rates than does PSF with instrumentation only $[12,15,16,18,19]$. Yaszay et al. [15] conducted a multicenter, retrospective study and reported that posterior spinal correction and fusion without resection of the hemivertebra had a lower complication rate than did in situ fusion and hemivertebrectomy. They noted that these patients were older and had larger secondary curves, where correction via hemivertebrectomy may lead to an imbalance in the spine. Lee et al. [18] had reported that PSF with instrumentation had a significantly lower operative time and less blood loss; however, their selection of correction technique was based on intraoperative flexibility and assessment. In both studies, no detailed selection criteria have been described to guide the selection of surgical methods.

Thus, this study was conducted to determine the perioperative and radiological outcomes of single-stage posterior passive correction and fusion (SSPPCF) using a pedicle screw system in adolescent patients who presented with congenital scoliosis with definitive preoperative selection criteria set by the University of Malaya Medical Centre.

\section{Materials and Methods}

\section{Patients}

This was a retrospective review of patients with congenital scoliosis who underwent SSPPCF using a pedicle screw system from 2008 to 2018 at a single institution. The institutional review board of the University of Malaya Medical Centre approved the study (MECID no., 201626-4669), and patient consent was obtained for publication. The medical records, plain radiographs, computed tomography scans, and magnetic resonance imaging images were evaluated.

The following inclusion criteria were applied: (1) adolescent patients aged $\geq 10$ years; (2) adolescent patients with adequate maturity for spinal fusion, as indicated by a closed triradiate cartilage; (3) main curve with Cobb angle of $>40^{\circ}$; and (4) patients who had SSPPCF because of one of the following indications: (a) patients with hemivertebra or wedge vertebra over the thoracic or thoracolumbar region with structural lumbar curves (supine side bending $\geq 25^{\circ}$ ) (Figs. 1, 2); (b) patients with hemivertebra or wedge vertebra at the lumbar region with significant pelvic obliquity or sacral slanting (Figs. 3, 4); and (c) patients with mixed or complex congenital scoliosis (Fig. 5).

The following exclusion criteria were applied: (1) patients who had previously undergone spinal fusion surgery with or without instrumentation, (2) patients who had undergone anterior spinal surgery, (3) patients who had incomplete medical records, and (4) patients who did not appear for each follow-up.

The lesions were classified according to the classifications proposed by Winter et al. [20] and Nasca et al. [21] and modified by McMaster and Ohtsuka [22]. Tables 1 and 2 list the data collected.

\section{Variables}

The calculated outcome parameters are defined below:

1) Estimated total blood loss

Total blood loss was estimated from the cell salvage system using the following formula: total blood loss $(\mathrm{mL})=($ final volume accumulated in the reservoir $)-($ total volume of anticoagulant citrate dextrose $[\mathrm{ACD}])-$ (total irrigation fluid used intraoperatively)+(total unfiltered blood). The total volume of ACD and unfiltered blood (the 

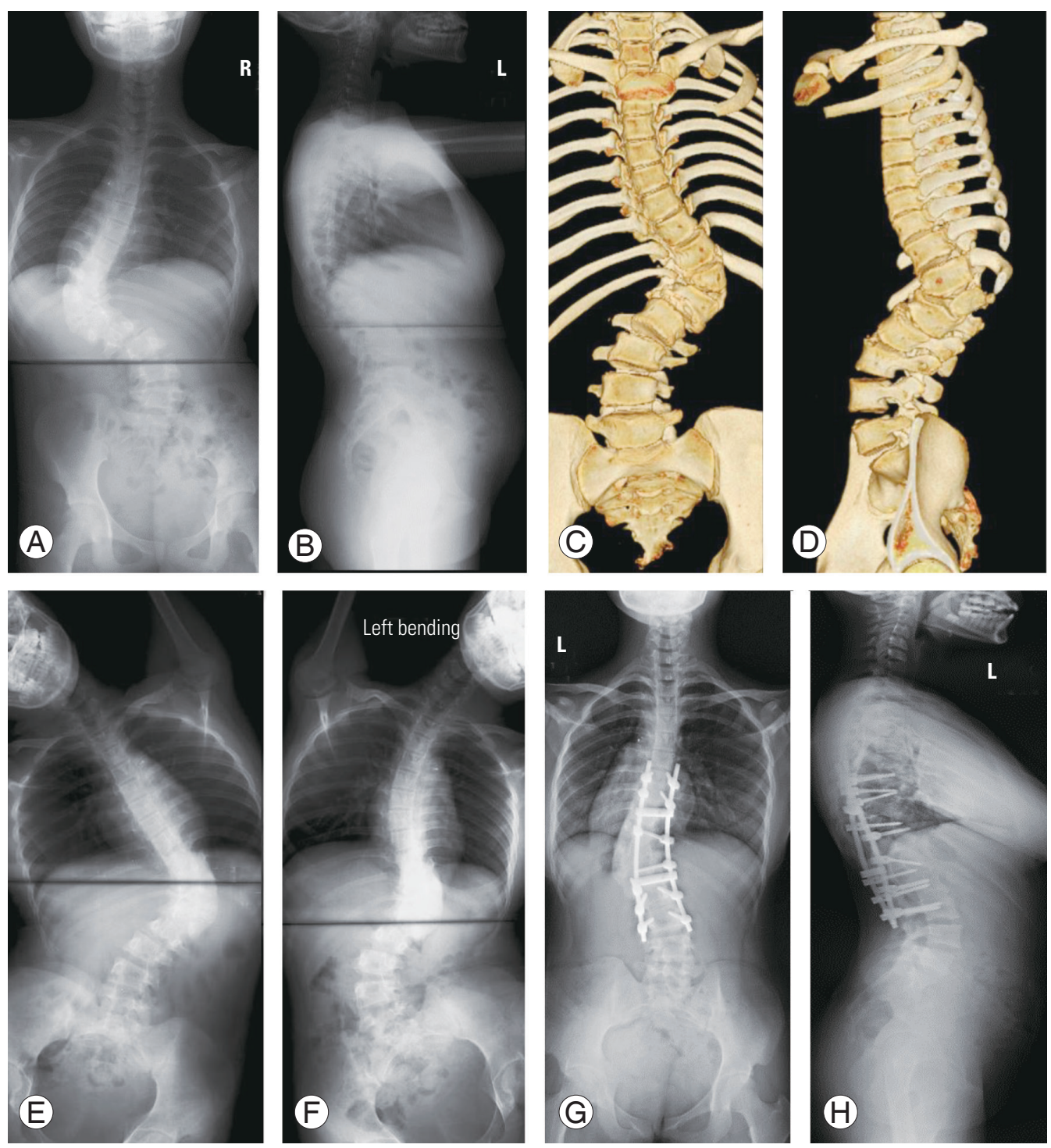

Fig. 1. Preoperative posteroanterior (A) and lateral (B) plain radiographs and three-dimensional computed tomography reconstruction films (C, D) of a patient with L1 hemivertebra with late structural changes in the lumbar spine and coronal imbalance. (E, F) Right and left supine side bending radiographs were shown in (E) and (F), respectively. The patient underwent single stage posterior passive correction and fusion. (G, H) Postoperative radiographs at 2-year follow-up showed good coronal balance.

difference between weights of used and dry reservoirs) was calculated, whereas the total irrigation fluid was measured intraoperatively. Total blood loss excluded the volume of postoperative drainage from the subfascial drain.

\section{2) Transfusion threshold}

The transfusion threshold was hemoglobin level $<8 \mathrm{~g} / \mathrm{dL}$ or when the patient was symptomatic.

3) Cobb angle correction rate

Cobb angle correction rate (CR) was calculated using the
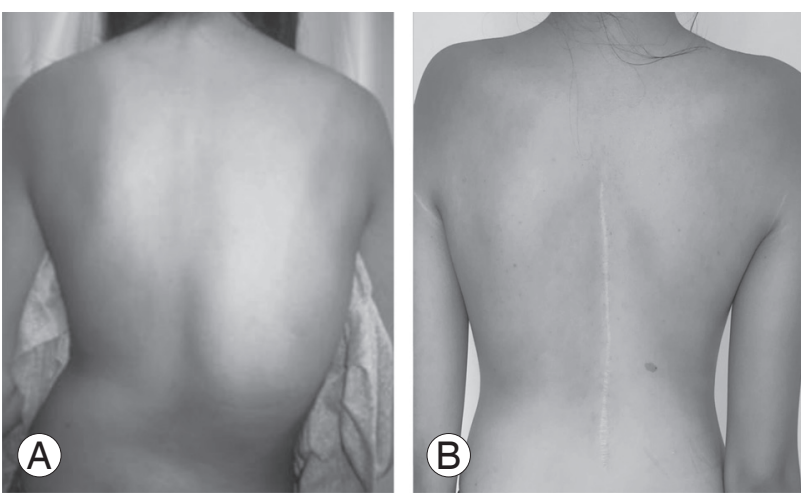

Fig. 2. Preoperative (A) and postoperative (B) clinical picture of patient in Fig. 1. 

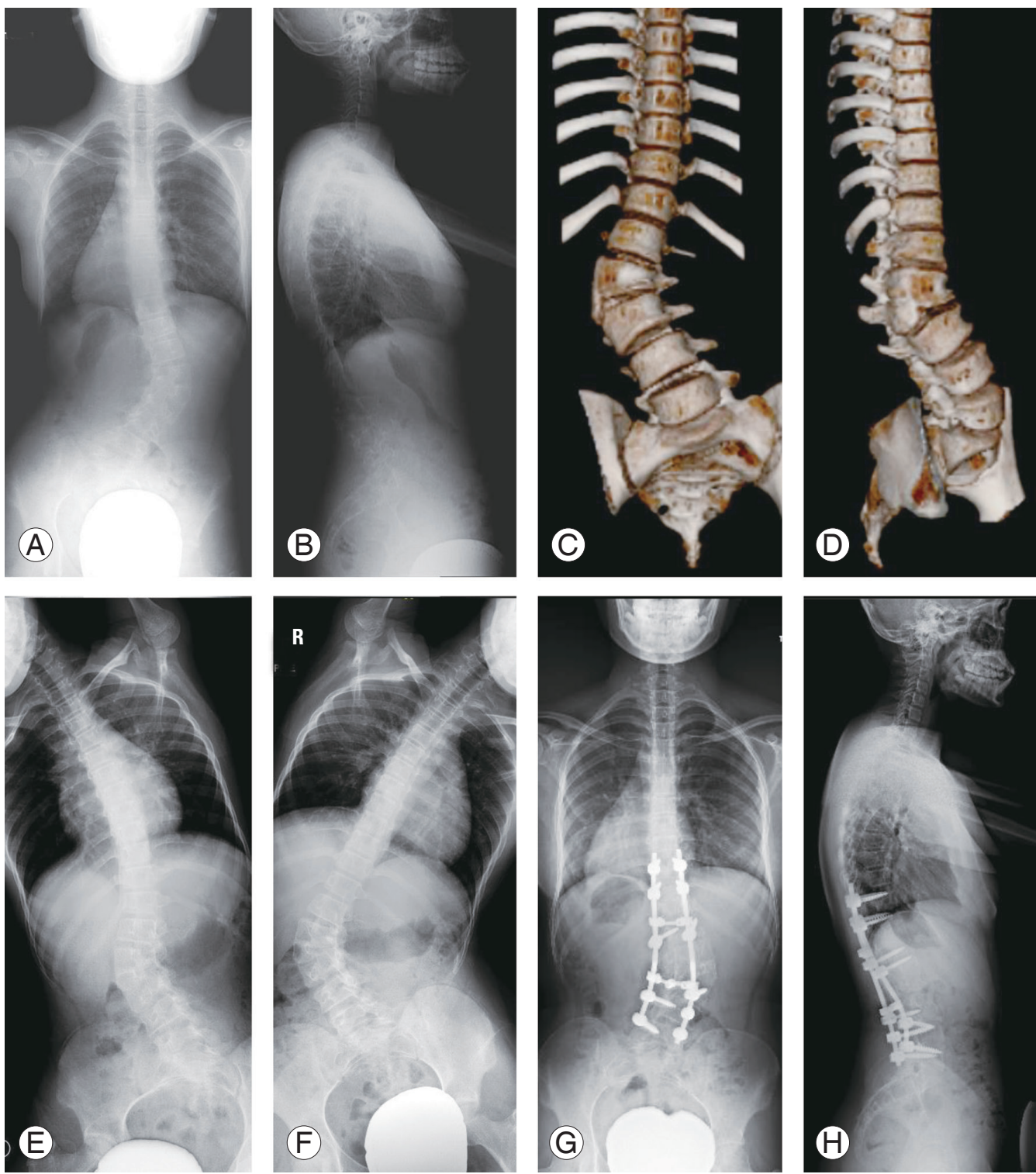

Fig. 3. Preoperative posteroanterior (A) and lateral (B) plain radiographs and three-dimensional computed tomography reconstruction films (C, D) of a patient with L3 wedged vertebra with pelvic obliquity and coronal imbalance. (E, F) Right and left supine side bending radiographs were shown in $\mathrm{E}$ and $\mathrm{F}$, respectively. The patient underwent single stage posterior passive correction and fusion. (G, H) Postoperative radiographs at 2-year follow-up showed good coronal balance.
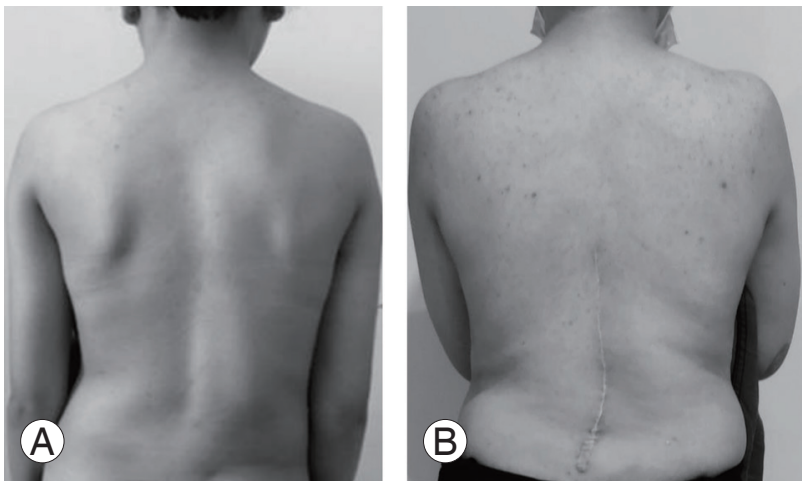

following formula: [(preoperative major Cobb angle-postoperative major Cobb angle)/preoperative major Cobb angle] $\times 100$.

\section{4) Regional kyphotic correction rate}

Regional kyphotic CR was calculated using the following formula: [(preoperative regional kyphotic angle-postoperative regional kyphotic angle)/preoperative regional kyphotic angle] $\times 100$.

Fig. 4. Preoperative (A) and postoperative (B) clinical picture of patient in Fig. 3. 

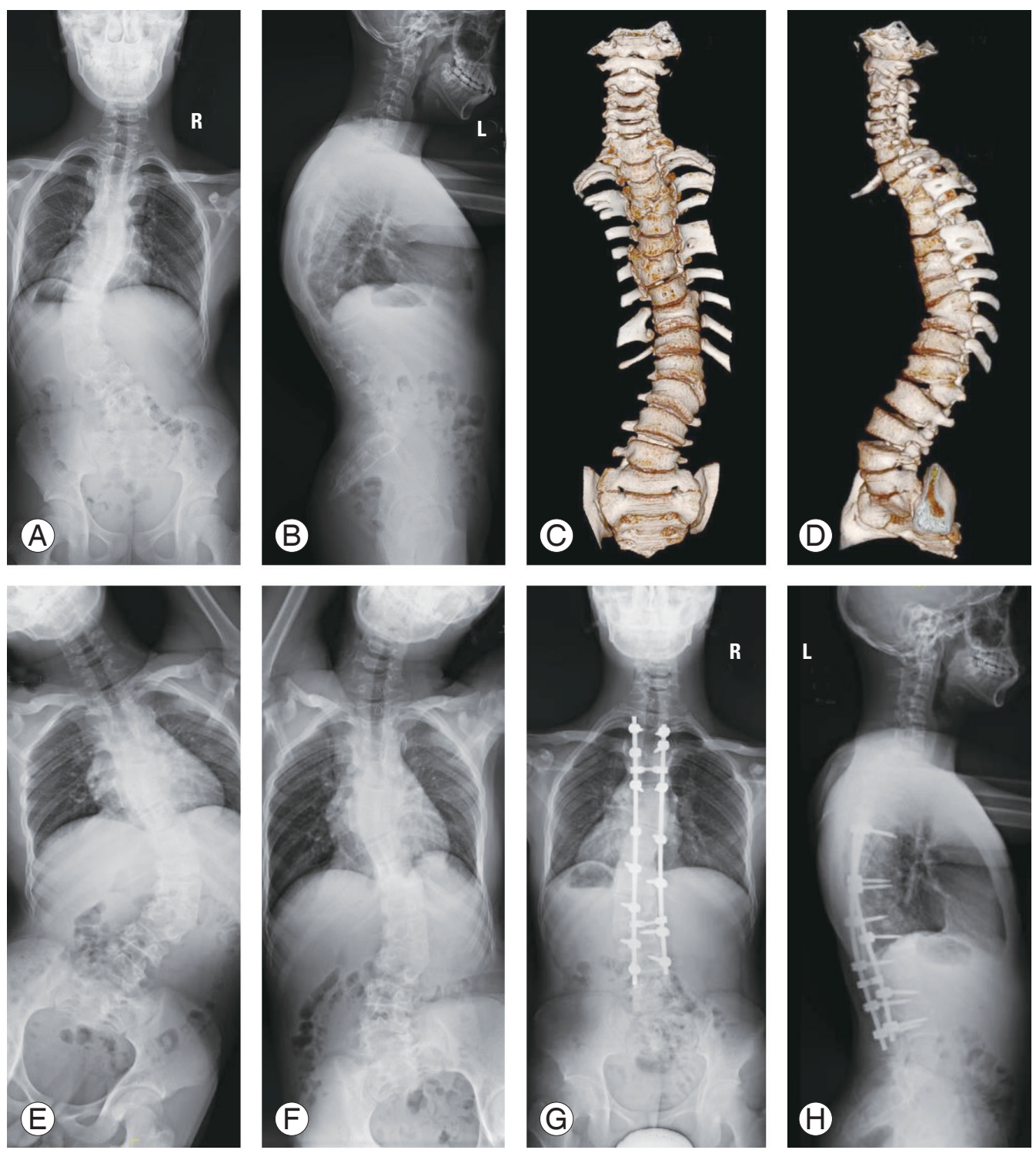

Fig. 5. Preoperative posteroanterior (A) and lateral (B) plain radiographs and three-dimensional computed tomography reconstruction films (C, D) of a patient with complex congenital spinal deformity including multilevel unsegmented vertebrae, hemivertebra and wedged vertebra. (E, F) Right and left supine side bending radiographs were shown in $E$ and $F$, respectively. The patient underwent single stage posterior passive correction and fusion. (G, H) Postoperative radiographs at 2-year follow-up showed good coronal balance.

\section{Preoperative assessment}

All patients underwent erect posteroanterior and lateral whole spine radiography. Flexibility assessment was performed with supine side-bending film, performed by an orthopedic resident or fellow who had received instructions and training for this procedure under expert supervision (Fig. 6). Trunk bending was performed passively to achieve maximum trunk bending while ensuring the absence of pelvic rotation. The patient was then instructed to maintain the position while the radiograph was captured.
Right- and left-side bending films were performed. Moreover, fulcrum-lateral and fulcrum supine-bending radiographs were obtained to assess the flexibility of kyphotic deformity (Fig. 7).

\section{Operative procedure}

All operations were performed by senior surgeons. Intravenous tranexamic acid was administered to all patients before skin incision. Spinal cord monitoring was performed during surgery. The lowest instrumented verte- 

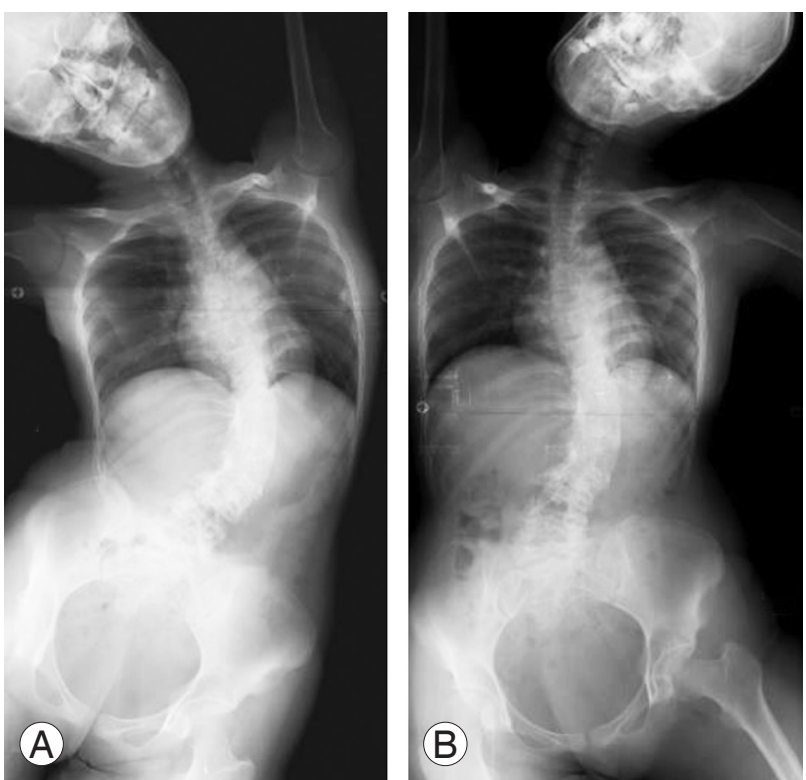

Fig. 6. Flexibility assessment radiographs with right supine side bending (A) and left supine side bending films (B)

bra was chosen based on the last vertebra substantially touched by the central sacral vertical line. Selection of the upper instrumented vertebra (UIV) was performed, referring to the supervised supine side-bending radiographs [23] (Fig. 6). These side-bending radiographs were used to calculate the optimal UIV tilt angle. The selected UIV was the most distal vertebra with a favorable UIV tilt angle and good proximal compensatory ability. Three or four pedicle screws were inserted over the proximal and distal foundation. An alternate level screw-placement strategy was used in between. Additional screws were inserted wherever necessary to achieve additional correction. Pedicle screws were inserted using the free-hand technique. Radical release included complete excision of the inferior articular facet, spinous processes, inferior part of the lamina, and thinning of the ligamentum flavum. Ponte osteotomies were performed when kyphosis correction was required. The correction was performed using a rodtranslation technique or cantilever method. The amount of correction targeted was based on the preoperative calculation of the UIV tilt angle [23,24]. This was confirmed intraoperatively with the crossbar method [25]. Fusion was augmented using autogenous bone grafts harvested from the spinous processes, laminae, transverse processes, and facet joints. Cell Saver 5+ Autologous Blood Recovery System (Haemonetics Corp., Braintree, MA, USA) was the cell salvage system used for all patients. A subfascial
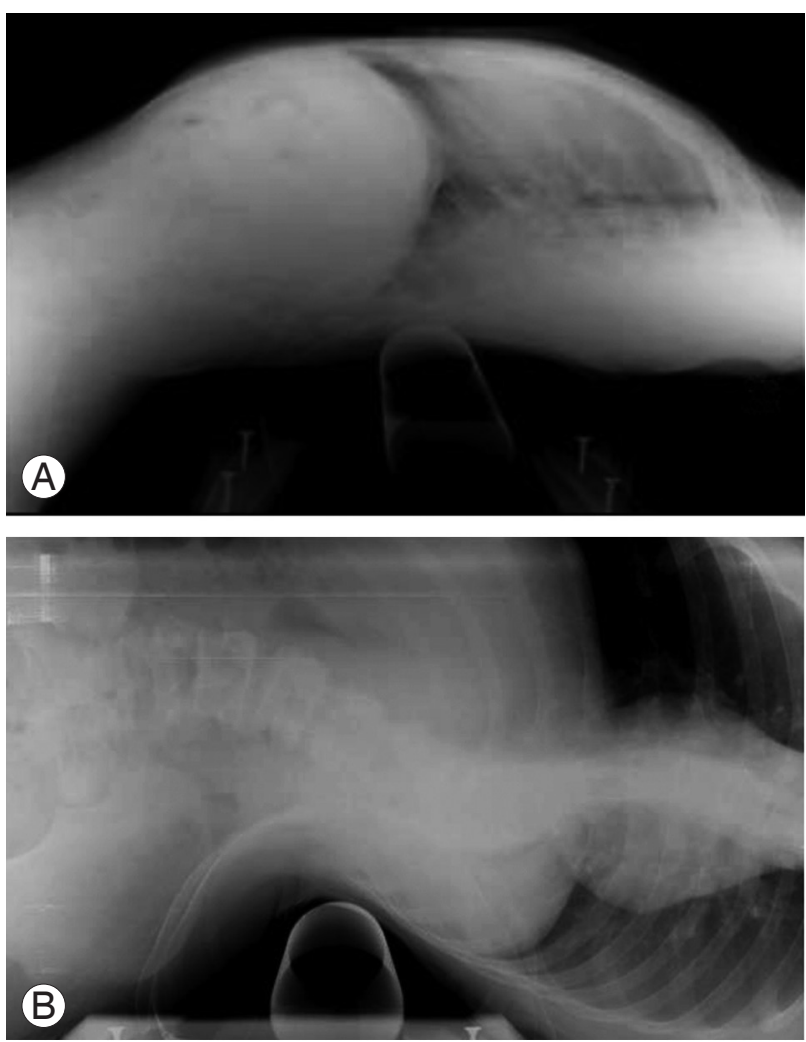

Fig. 7. Fulcrum bending radiographs—-supine lateral ( $\mathbf{A}$ ) and lateral posteroanterior (B) — used to assess the flexibility of the kyphotic and scoliotic deformity.

drain was inserted, and subcutaneous bupivacaine was infiltrated before wound closure.

\section{Statistical analyses}

All data were analyzed using IBM SPSS ver. 25.0 (IBM Corp., Armonk, NY, USA). Demographic variables were analyzed using descriptive statistics, whereas continuous variables were analyzed using paired $t$-test to investigate the differences in preoperative and postoperative radiographic parameters. All $p$-values were two-tailed, and a $p$-value of $<0.05$ was considered to be statistically significant.

\section{Results}

\section{Main results}

Thirty-four patients with congenital scoliosis who underwent SSPPCF from 2008 to 2018 were enrolled in the study. The study population included four males and 

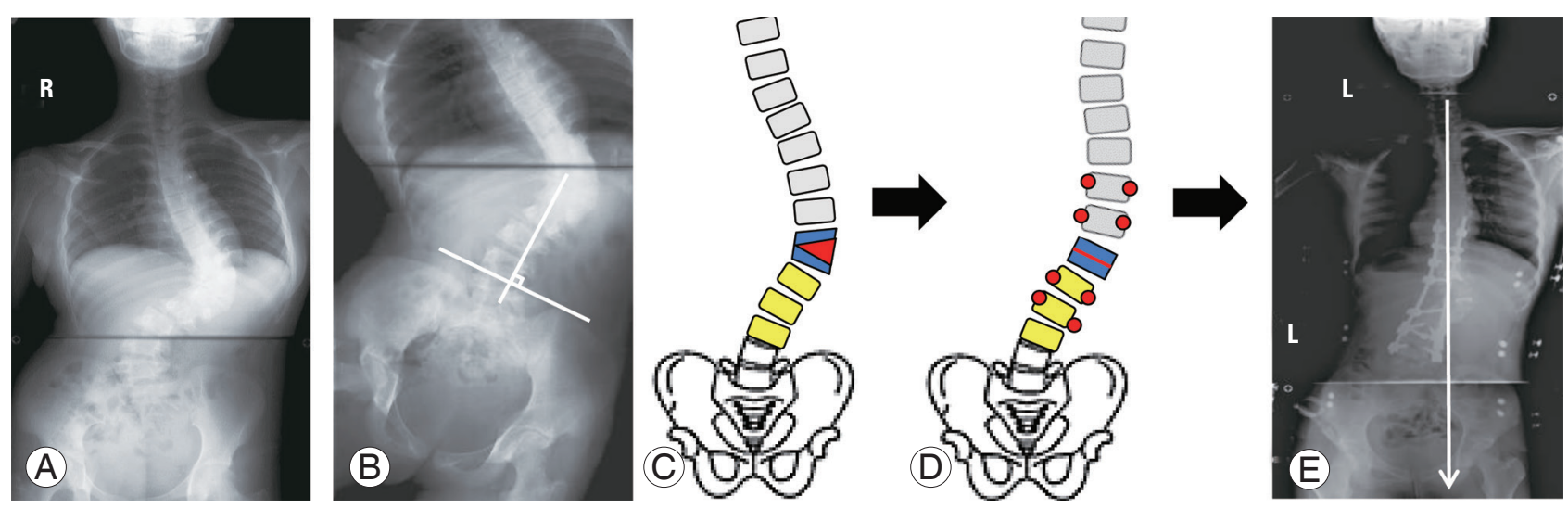

Fig. 8. (A-E) Illustration on how the excision of hemivertebra in a patient with structural lumbar curve may lead to coronal imbalance.

30 females. The follow-up duration was $12-120$ months with an average follow-up duration of $43.6 \pm 32.9$ months. Table 1 shows the demographic and radiographic data. The average patient age was $14.6 \pm 3.4$ years at the time of surgery. The mean patient height was $148.9 \pm 10.4 \mathrm{~cm}$, mean weight was $46.0 \pm 11.5 \mathrm{~kg}$, and mean body mass index was $20.9 \pm 6.0 \mathrm{~kg} / \mathrm{m}^{2}$. There were 13 hemivertebrae, three wedged vertebrae, two butterfly vertebrae, three hemivertebrae with butterfly vertebra, eight unsegmented bars, and five multiple complex lesions. The location of the hemivertebra was noted on the T6-L3 region (most common at T11-L2), hemivertebra with butterfly vertebra on the main thoracic region, unsegmented bar at T3-T10, and wedged vertebra over T11, L3, and L5. Five patients underwent SSPPCF for complex lesions that include two low lumbar hemivertebrae, one L5 wedged vertebra, and one thoracolumbar lesion, mostly due to hemivertebra. Seven patients had neuroaxial anomalies (four syringomyelia, two diastematomyelia, and one tethered cord anomaly). Patients with neuroaxial anomalies were referred for neurosurgical intervention or clearance before spine surgeries.

Table 1 shows the perioperative data. The average blood loss was 1,208.4 $\pm 763.5 \mathrm{~mL}$ (range, 277-3,000 mL). Seven patients $(20.6 \%)$ needed allogeneic blood transfusions. The average length of hospital stay was $6.1 \pm 2.5$ days. The complication rate was $11.8 \%$, and the following four complications were noted: severe intraoperative blood loss in one patient, rod breakage in one patient, and distal adding-on in two patients. The patient with severe intraoperative blood loss was a 16-year-old female patient who had thalassemia major with L1 hemivertebra. She had 3,000 $\mathrm{mL}$ blood loss and underwent blood transfusion. She re- covered well and was discharged on postoperative day 5 . After 1 year, she was noted to have one rod breakage. She was advised to undergo revision surgery but opted to undergo observation.

Table 2 shows the comparison between preoperative and postoperative radiographic parameters. All the coronal radiological parameters, the Cobb angle, radiographic shoulder height, clavicle angle, T1 tilt, cervical axis, pelvic obliquity coronal balance, and apical vertebral translation showed significant $(p<0.05)$ improvement postoperatively. Postoperatively, the Cobb angle reduced significantly from $65.9^{\circ} \pm 17.4^{\circ}$ to $36.3^{\circ} \pm 15.3^{\circ}(p<0.001)$. For sagittal parameters, no significant worsening was noted. Regional kyphotic angle reduced significantly from $39.9^{\circ} \pm 20.5^{\circ}$ to $27.5^{\circ} \pm 13.9^{\circ}(p=0.001)$. The Cobb angle CR was $44.8 \% \pm 17.4 \%$, and the regional kyphotic angle CR was $19.3 \% \pm 49.6 \%$.

\section{Case illustrations on each surgical indication for SSPPCF}

1) Patient 1: hemivertebra or wedge vertebra over the thoracic or thoracolumbar region with structural lumbar curves (supine side bending $\geq 25^{\circ}$ ).

A 13-year-old girl presented with late structural changes in the lumbar spine and coronal imbalance caused by L1 hemivertebra. Preoperative coronal balance was $>20 \mathrm{~mm}$. The surgical duration was 170 minutes. Estimated blood loss was $900 \mathrm{~mL}$, and the duration of hospital stay was 5 days. Postoperative radiographs obtained at the 2-year follow-up showed good coronal balance $(-5 \mathrm{~mm})$ (Figs. 1, 2).

2) Patient 2: hemivertebra or wedge vertebra at the lumbar region with significant pelvic obliquity or sacral slanting 
Table 1. Demographic, radiographic, and perioperative data

\begin{tabular}{|c|c|}
\hline Characteristic & Value \\
\hline \multicolumn{2}{|l|}{ Demographic data } \\
\hline Age (yr) & $14.6 \pm 3.4$ \\
\hline \multicolumn{2}{|l|}{ Gender } \\
\hline Female & $30(88.2)$ \\
\hline Male & $4(11.8)$ \\
\hline Height (cm) & $148.9 \pm 10.4$ \\
\hline Weight (kg) & $46.0 \pm 11.5$ \\
\hline Body mass index $\left(\mathrm{kg} / \mathrm{m}^{2}\right)$ & $20.9 \pm 6.0$ \\
\hline \multicolumn{2}{|l|}{ Preoperative radiographic data } \\
\hline \multicolumn{2}{|l|}{ Types of anomalies } \\
\hline Hemivertebra & $13(38.2)$ \\
\hline Wedged vertebra & $3(8.8)$ \\
\hline Butterfly vertebra & $2(5.9)$ \\
\hline Hemivertebra with butterfly vertebra & $3(8.8)$ \\
\hline Unsegmented bar & $8(23.5)$ \\
\hline Complex & $5(14.8)$ \\
\hline Neuroaxial anomalies & $7(20.6)$ \\
\hline Syringomyelia & $4(11.8)$ \\
\hline Diastematomyelia & $2(5.9)$ \\
\hline Tethered cord & $1(2.9)$ \\
\hline Cobb angle $\left({ }^{\circ}\right)$ & $65.9 \pm 17.4$ \\
\hline Regional kyphotic angle $\left({ }^{\circ}\right)$ & $39.9 \pm 20.5$ \\
\hline \multicolumn{2}{|l|}{ Perioperative data } \\
\hline Operation duration (min) & $219.4 \pm 68.8$ \\
\hline No. of fused levels & $11.1 \pm 2.9$ \\
\hline No. of screws & $13.3 \pm 2.4$ \\
\hline Blood loss (mL) & $1,208.4 \pm 763.5$ \\
\hline Preoperative hemoglobin (g/L) & $13.2 \pm 1.6$ \\
\hline Postoperative day 2 hemoglobin (g/L) & $10.8 \pm 2.0$ \\
\hline Transfusion rate & $7(20.6)$ \\
\hline Length of hospital stay (day) & $6.1 \pm 2.5$ \\
\hline Complications & $4(11.8)$ \\
\hline Severe intraoperative blood loss & $1(2.9)$ \\
\hline Rod breakage & $1(2.9)$ \\
\hline Distal adding-on & $2(5.9)$ \\
\hline
\end{tabular}

Values are presented as mean \pm standard deviation or number (\%).

A 14-year-old girl complained of a "slanted posture" caused by scoliosis with L3 wedged vertebra. Preoperative coronal balance was $32 \mathrm{~mm}$. The surgical duration was 130 minutes, estimated blood loss was $500 \mathrm{~mL}$, and hospital stay was 5 days. Postoperative radiographs obtained at the 2 -year
Table 2. Preoperative and postoperative radiographic parameters

\begin{tabular}{|c|c|c|c|}
\hline Variable & Preoperative & Postoperative & $p$-value \\
\hline \multicolumn{4}{|l|}{ Coronal parameters } \\
\hline Cobb angle $\left({ }^{\circ}\right)$ & $65.9 \pm 17.4$ & $36.3 \pm 15.3$ & $<0.001^{*}$ \\
\hline $\begin{array}{l}\text { Radiographic shoulder } \\
\text { height }(\mathrm{mm})\end{array}$ & $13.5 \pm 12.3$ & $5.8 \pm 7.5$ & $0.001^{*}$ \\
\hline Clavicle angle $\left({ }^{\circ}\right)$ & $4.7 \pm 6.8$ & $1.5 \pm 1.8$ & $0.019^{*}$ \\
\hline T1 tilt $\left({ }^{\circ}\right)$ & $10.1 \pm 9.5$ & $5.5 \pm 7.1$ & $<0.001^{*}$ \\
\hline Cervical axis $\left({ }^{\circ}\right)$ & $4.0 \pm 5.0$ & $2.1 \pm 3.7$ & $0.001^{*}$ \\
\hline Pelvic obliquity $\left({ }^{\circ}\right)$ & $3.7 \pm 5.0$ & $1.8 \pm 2.0$ & $0.021^{*}$ \\
\hline Coronal balance (mm) & $16.9 \pm 13.2$ & $5.2 \pm 5.1$ & $<0.001^{*}$ \\
\hline $\begin{array}{l}\text { Apical vertebral translation } \\
(\mathrm{mm})\end{array}$ & $50.9 \pm 17.9$ & $24.8 \pm 8.1$ & $<0.001^{*}$ \\
\hline \multicolumn{4}{|l|}{ Sagittal parameters } \\
\hline Regional kyphotic angle $\left(^{\circ}\right)$ & $39.9 \pm 20.5$ & $27.5 \pm 13.9$ & $0.001^{*}$ \\
\hline T5-T12 kyphosis $\left({ }^{\circ}\right)$ & $24.7 \pm 27.6$ & $23.3 \pm 13.8$ & 0.580 \\
\hline Sagittal vertical axis (mm) & $-4.2 \pm 30.7$ & $-2.5 \pm 32.4$ & 0.602 \\
\hline \multicolumn{4}{|l|}{ Correction rate (\%) } \\
\hline Cobb angle correction rate & & $44.8 \pm 17.4$ & \\
\hline $\begin{array}{l}\text { Regional kyphotic angle } \\
\text { correction rate }\end{array}$ & & $19.3 \pm 49.6$ & \\
\hline
\end{tabular}

Values are presented as mean \pm standard deviation.

${ }^{*} \mathrm{~A} p$-value is statistically significant.

follow-up were coronally balanced ( $0 \mathrm{~mm}$ ) (Figs. 3,4$)$.

3) Patient 3: mixed or complex congenital scoliosis

A 14-year-old boy presented with complex spinal deformity with multilevel unsegmented vertebrae, hemivertebrae, and wedged vertebrae. The surgical duration was 280 minutes, estimated blood loss was 1,100 mL, and hospital stay duration was 7 days. Postoperative radiographs obtained at the 2-year follow-up showed adequate balance (Fig. 5).

\section{Discussion}

A patient with congenital scoliosis in adolescence presents for spine surgery because of several reasons, including parental decision to wait, failure of brace treatment, missed or late diagnosis, or delay due to primary treatment of neuroaxial pathologies. Surgical treatment for these patients remains complex. In skeletally matured patients with congenital scoliosis, compensatory secondary curves may be larger and less flexible [15]. This obviates the feasibility of a hemivertebrectomy that produces a large correction at the main deformity leading to an imbalance 
body, arising from the residual deformity of the secondary curves. Although a hemivertebrectomy was performed, long fusion may still be required to include the correction of the secondary curves to balance the body [13]. Thus, hemivertebrectomy is generally recommended for younger patients with flexible spine before the development of structural secondary curves [13-15]. Logically, this also applies to other procedures that osteotomize the congenital anomaly for large corrections, such as PSOs, wedge osteotomies, and VCRs. In contrast, spinal balance can be achieved more easily than with PSF without osteotomies because the correction of the main congenital deformity is less, making the correction of the secondary curves easier to achieve a balanced spine.

We identified three strong indications for SSPPCF. The first indication was hemivertebra or wedge vertebra over the thoracic or thoracolumbar region with structural lumbar curves (supine side bending $\geq 25^{\circ}$ ) (Figs. 1, 2). These patients exhibited stiff secondary lumbar curves, wherein the extension of the correction and fusion into the lumbar region was necessary. If the excision of the hemivertebra was performed, it would potentially cause a postoperative coronal imbalance of the body, wherein a revision to an even longer fusion would be needed (Fig. 8). The second indication was hemivertebra or wedge vertebra at the lumbar region with significant pelvic obliquity or sacral slanting (Figs. 3, 4). Careful preoperative flexibility assessment should be performed for these patients because their lumbosacral junction may be stiff, disallowing the return of the lumbar spine to the midline. In several cases, fusion to the sacrum may be needed. Similarly, if the excision of the hemivertebra was performed, the risk of coronal decompensation would be much higher. However, by performing SSPPCF, it is easier to achieve spinal balance and potentially, without the need for fusion to the sacrum. The third indication was patients with mixed or complex congenital scoliosis (Fig. 5). In these patients, SSPPCF may be safer and less invasive than procedures needing osteotomies.

In this study, the average surgical duration was $219.4 \pm 68.8$ minutes and the average blood loss was approximately $1,208.4 \pm 763.5 \mathrm{~mL}$. Seven patients $(20.6 \%)$ needed blood transfusion. The average length of hospital stay was $6.1 \pm 2.5$ days. The complication rate was $11.8 \%$, with one patient exhibiting severe blood loss, one patient with a single rod breakage, and two patients with distal adding-on (Table 1). Yaszay et al. [15] reported an average surgical duration of $324 \pm 124$ minutes and average blood loss of $837 \pm 692 \mathrm{~mL}$; Lee et al. [18] reported an average surgical duration of 308.14 minutes and average blood loss of $540.06 \mathrm{~mL}$; in comparison, our patients had shorter surgical duration but higher intraoperative blood loss. The complication rate in our trial was lower than that reported by Yaszay et al. [15] (15\%). With respect to radiological parameters, all coronal and sagittal parameters showed improvement in the Cobb angle, radiographic shoulder height, clavicle angle, T1 tilt, cervical axis, pelvic obliquity, coronal balance, and apical vertebral translation, and RKA showed significant $(p<0.05)$ improvement postoperatively (Table 2). Patients achieved a coronal Cobb angle CR of $44.8 \% \pm 17.4 \%$ and an RKA CR of $19.3 \% \pm 49.6 \%$. This was comparable with that reported by Yaszay et al. [15]; they reported a coronal Cobb angle CR of approximately $40 \%$ and a sagittal Cobb angle CR of approximately $27 \%$. Lee et al. [18] reported a coronal Cobb angle CR of approximately $60.27 \%$ and a kyphosis CR of approximately $10 \%$.

There were certain limitations of this study. The study sample size was relatively small; thus, the statistical strength of the study was reduced. We did not assess the health-related quality of life scores for the patients to evaluate their postoperative clinical outcomes. The types of lesions in congenital scoliosis were diverse, and it was difficult to standardize a specific surgical strategy for each patient. However, a general approach was used to make the decision regarding the type of surgery to be performed, leaving the specific surgical management individually tailored to each patient. SSPPCF has been reported in previous studies and is a widely accepted surgical option, and our results further support this surgical technique. The surgical indications suggested in this manuscript are based on the senior authors' expert opinion, and further validation studies may be required to confirm the accuracy of the present findings.

\section{Conclusions}

To conclude, SSPPCF provided a significant coronal spinal correction to attain a more balanced spine. It was a feasible option for adolescent patients with congenital scoliosis. With SSPPCF, the presence of stiffer secondary curves, especially at the lumbar spine and lumbosacral junction, obviates the need for osteotomy procedures. 


\section{Conflict of Interest}

No potential conflict of interest relevant to this article was reported.

\section{References}

1. Tikoo A, Kothari MK, Shah K, Nene A. Current concepts: congenital scoliosis. Open Orthop J 2017;11:337-45.

2. Hedequist DJ. Surgical treatment of congenital scoliosis. Orthop Clin North Am 2007;38:497-509.

3. Arlet V, Odent T, Aebi M. Congenital scoliosis. Eur Spine J 2003;12:456-63.

4. Sponseller PD, Ting BL. Congenital scoliosis. In: Herkowitz HN, Garfin SR, Eismont FJ, Bell GR, Balderston RA, editors. Rothman-Simeone: the spine. 6th ed. Philadelphia (PA): Saunders Elservier; 2011. p. 374-84.

5. Winter RB. Congenital thoracic scoliosis with unilateral unsegmented bar, convex hemivertebrae, and fused concave ribs with severe progression after posterior fusion at age 2: 40-year follow-up after revision anterior and posterior surgery at age 8 . Spine (Phila Pa 1976) 2012;37:E507-10.

6. Kesling KL, Lonstein JE, Denis F, et al. The crankshaft phenomenon after posterior spinal arthrodesis for congenital scoliosis: a review of 54 patients. Spine (Phila Pa 1976) 2003;28:267-71.

7. Cheung KM, Zhang JG, Lu DS, K Luk KD, Y Leong JC. Ten-year follow-up study of lower thoracic hemivertebrae treated by convex fusion and concave distraction. Spine (Phila Pa 1976) 2002;27(7):748-53.

8. Winter RB, Lonstein JE, Denis F, Sta-Ana de la Rosa $\mathrm{H}$. Convex growth arrest for progressive congenital scoliosis due to hemivertebrae. J Pediatr Orthop 1988;8:633-8.

9. Campbell RM Jr, Hell-Vocke AK. Growth of the thoracic spine in congenital scoliosis after expansion thoracoplasty. J Bone Joint Surg Am 2003;85:409-20.

10. Yazici M, Emans J. Fusionless instrumentation systems for congenital scoliosis: expandable spinal rods and vertical expandable prosthetic titanium rib in the management of congenital spine deformities in the growing child. Spine (Phila Pa 1976) 2009;34:1800-7.

11. Pahys JM, Guille JT. What's new in congenital scoliosis? J Pediatr Orthop 2018;38:e172-9.
12. Li XF, Liu ZD, Hu GY, et al. Posterior unilateral pedicle subtraction osteotomy of hemivertebra for correction of the adolescent congenital spinal deformity. Spine J 2011;11:111-8.

13. Ruf M, Harms J. Posterior hemivertebra resection with transpedicular instrumentation: early correction in children aged 1 to 6 years. Spine (Phila Pa 1976) 2003;28:2132-8.

14. Ruf M, Jensen R, Letko L, Harms J. Hemivertebra resection and osteotomies in congenital spine deformity. Spine (Phila Pa 1976) 2009;34:1791-9.

15. Yaszay B, O'Brien M, Shufflebarger HL, et al. Efficacy of hemivertebra resection for congenital scoliosis: a multicenter retrospective comparison of three surgical techniques. Spine (Phila Pa 1976) 2011;36:2052-60.

16. Helenius I, Serlo J, Pajulo O. The incidence and outcomes of vertebral column resection in paediatric patients: a population-based, multicentre, follow-up study. J Bone Joint Surg Br 2012;94:950-5.

17. Matsumoto M, Watanabe K, Hosogane N, Toyama Y. Updates on surgical treatments for pediatric scoliosis. J Orthop Sci 2014;19:6-14.

18. Lee CS, Hwang CJ, Kim DJ, et al. Feasibility of correction with instrumentation only in congenital scoliosis. Neurosurgery 2014;74:35-41.

19. Reames DL, Smith JS, Fu KM, et al. Complications in the surgical treatment of 19,360 cases of pediatric scoliosis: a review of the Scoliosis Research Society Morbidity and Mortality Database. Spine (Phila Pa 1976) 2011;36:1484-91.

20. Winter RB, Moe JH, Eilers VE. Congenital scoliosis a study of 234 patients treated and untreated: part I: natural history. J Bone Joint Surg 1968;50:1-5.

21. Nasca RJ, Stilling FH 3rd, Stell HH. Progression of congenital scoliosis due to hemivertebrae and hemivertebrae with bars. J Bone Joint Surg Am 1975;57:456-66.

22. McMaster MJ, Ohtsuka K. The natural history of congenital scoliosis: a study of two hundred and fiftyone patients. J Bone Joint Surg Am 1982;64:1128-47.

23. Kwan MK, Chan CY. Is there an optimal upper instrumented vertebra (UIV) tilt angle to prevent postoperative shoulder imbalance and neck tilt in Lenke 1 and 2 adolescent idiopathic scoliosis (AIS) patients? Eur Spine J 2016;25:3065-74.

24. Kwan MK, Chiu CK, Chan TS, Abd Gani SM, Tan $\mathrm{SH}$, Chan CY. Flexibility assessment of the unfused 
thoracic segments above the "potential upper instrumented vertebrae" using the supine side bending radiographs in Lenke 5 and 6 curves for adolescent idiopathic scoliosis patients. Spine J 2018;18:53-62.
25. Kwan MK, Chiu CK, Goh SH, et al. The reliability of intraoperative crossbar technique in determining the upper instrumented vertebra (UIV) tilt angle for adolescent idiopathic scoliosis (AIS) undergoing posterior spinal fusion. Clin Spine Surg 2019;32:256-62. 